\title{
HODGE THEORY AND DEFORMATIONS OF AFFINE CONES OF SUBCANONICAL PROJECTIVE VARIETIES
}

\author{
CARMELO DI NATALE, ENRICO FATIGHENTI, AND DOMENICO FIORENZA
}

\begin{abstract}
We investigate the relation between the Hodge theory of a smooth subcanonical $n$-dimensional projective variety $X$ and the deformation theory of the affine cone $A_{X}$ over $X$. We start by identifying $H_{\text {prim }}^{n-1,1}(X)$ as a distinguished graded component of the module of first order deformations of $A_{X}$, and later on we show how to identify the whole primitive cohomology of $X$ as a distinguished graded component of the Hochschild cohomology module of the punctured affine cone over $X$. In the particular case of a projective smooth hypersurface $X$ we recover Griffiths' isomorphism between the primitive cohomology of $X$ and certain distinguished graded components of the Milnor algebra of a polynomial defining $X$. The main result of the article can be effectively exploited to compute Hodge numbers of smooth subcanonical projective varieties. We provide a few example computation, as well a SINGULAR code, for Fano and Calabi-Yau threefolds.
\end{abstract}

\section{Contents}

1. Introduction

2. The Infinitesimal Deformation Module of an Affine Isolated Singularity

2.1. A Quick Review in Deformation Theory

2.2. $T^{1}$ and Hodge Theory

2.3. Obstructions and Automorphisms

2.4. A SINGULAR appendix: how to compute Hodge numbers using the $T^{i}$

3. Deformations of Derived Categories and Hodge Theory

3.1. A Primer on Noncommutative Schemes and Hochschild Structures

3.2. Hochschild Cohomology of Punctured Affine Cones

3.3. The case of a hypersurface

References

\section{INTRODUCTION}

Deformation Theory and Hodge Theory are known to be closely related. Examples of this friendship can be found, for example, in the theory of Variations of Hodge Structures, or in the Griffiths Residue Theory (see the original papers [21] or [35] for a more modern and detailed exposition). The latter identifies the Hodge structure of a smooth projective hypersurface with a subalgebra of the Milnor algebra of the hypersurface itself, an 
important deformation-theoretic invariant. More precisely, if

$$
X=\left\{\left[x_{0}, \ldots, x_{n+1}\right] \mid f\left(x_{0}, \ldots, x_{n+1}\right)=0\right\} \subseteq \mathbb{P}_{\mathbb{C}}^{n+1}
$$

is a smooth, degree $d, n$-dimensional projective hypersurface, Griffiths work establishes an isomorphism, given by a higher residue map

$$
H_{\text {prim }}^{p-1, n+1-p}(X) \cong\left(\mathcal{M}_{f}\right)_{p d-n-2} .
$$

The subscript prim in the above formula stands for primitive cohomology, and the object on the right is the degree $p d-n-2$ component of the Milnor algebra

$$
\mathcal{M}_{f}:=\mathbb{C}\left[x_{0}, \ldots, x_{n+1}\right] / J_{f},
$$

where $J_{f}=\left(\frac{\partial f}{\partial x_{0}}, \ldots, \frac{\partial f}{\partial x_{n+1}}\right)$, is the Jacobian ideal of $f$. The Milnor algebra $\mathcal{M}_{f}$ contains deformation data for $X$, in the sense that its degree $d$ component can be identified with the space of first order embedded deformations of $X$ in $\mathbb{P}_{\mathbb{C}}^{n+1}$.

Let us tackle the general case: from now on fix $X \subseteq \mathbb{P}_{\mathbb{C}}^{N}$ to be a smooth complex projective variety of $\operatorname{dim} X=n$ and arbitrary codimension in some projective space and consider the space of first order infinitesimal deformations of the affine cone $A_{X}$ of $X$. This space of first order deformations is classically known in the literature as $T_{A_{X}}^{1}$ (see [31] and [34]). It is a naturally graded vector space and in [31] Schlessinger shows that its degree 0 component is identified with the space of first order embedded deformations of the variety $X$ in $\mathbb{P}_{\mathbb{C}}^{N}$. When $X$ is a hypersurface, this module is nothing but the Milnor algebra of $X$ (up to a shift of degree $d$ ), but in general it is a much richer and more complicated object.

The superscript 1 in $T_{A_{X}}^{1}$ refers to the fact that this is indeed the degree 1 component of a graded module $T_{A_{X}}^{\bullet}$. As we will see, there are several possible definitions for this graded module, but under reasonable assumptions - for example, $X$ projectively normal - we can take as definition

$$
T_{A_{X}}^{i}:=\operatorname{Ext}_{\mathcal{O}_{A_{X}}}^{i}\left(\Omega_{A_{X}}^{1}, \mathcal{O}_{A_{X}}\right) .
$$

Here, $T_{A_{X}}^{1}$ represents - as already mentioned - first order deformations of $A_{X}$, while $T_{A_{X}}^{2}$ and $T_{A_{X}}^{0}$ encode respectively obstructions and infinitesimal automorphisms. It is important to notice that in this case - and more generally in the isolated-singularity case - both $T_{A_{X}}^{1}$ and $T_{A_{X}}^{2}$ will be finite-dimensional.

Now let us assume that the embedding $X \hookrightarrow \mathbb{P}_{\mathbb{C}}^{N}$ is subcanonical, i.e. that $\omega_{X} \cong \mathcal{O}_{X}(m)$ for some $m \in \mathbb{Z}$. Subcanonical varieties occupies a special case in literature: for a survey see for example [14], or [1]. Under this condition, we find an interesting generalization of Griffiths Residue Theorem. Our first results concern a deep relation between first order deformations, obstructions and automorphisms of $X$, and the Hodge theory of $X$ :

Theorem 1.1. Let $X$ be a smooth complex subcanonical projective variety of dimension $n$, and let $m \in \mathbb{Z}$ be the integer such that $\omega_{X} \cong \mathcal{O}_{X}(m)$.

(1) There is a natural isomorphism

$$
\left(T_{A_{X}}^{0}\right)_{m} \cong H_{\mathrm{prim}}^{n, 0}(X) .
$$


(2) If $H^{1}\left(X, \mathcal{O}_{X}(k)\right)=0$ for every $k \in \mathbb{Z}$, then there is a natural isomorphism

$$
\left(T_{A_{X}}^{1}\right)_{m} \cong H_{\text {prim }}^{n-1,1}(X) \text {. }
$$

(3) If also $H^{2}\left(X, \mathcal{O}_{X}(k)\right)=0$ for every $k \in \mathbb{Z}$, then there is a natural isomorphism

$$
\left(T_{A_{X}}^{2}\right)_{m} \cong H_{\text {prim }}^{n-2,1}(X) \text {. }
$$

Notice that the apparently quite restrictive conditions $H^{i}\left(X, \mathcal{O}_{X}(k)\right)=0$ for every $k \in \mathbb{Z}$ and for $i=1,2$ in the above theorem are actually satisfied by a large class of projective manifolds. Namely every arithmetically Cohen-Macaulay variety $X$ satisfies the vanishing condition for $i=1$ if $\operatorname{dim} X \geq 2$ and satisfies also the condition for $i=2$ if $\operatorname{dim} X \geq 3$.

The results stated in Theorem 1.1 have a surprising generalisation in terms of derived categories. It is well known (see [3]) that, if $X$ is Calabi Yau, there is an identification between the space of infinitesimal deformations of the derived category $D(X)$ and the Hodge Theory of $X$, given by the celebrated Hochschild-Kostant-Rosenberg isomorphism (see [12]).

The story is indeed famous in the literature: in the spirit of the work of Kontsevich [27], one first identifies the Hochschild cohomology of $X$ with the cohomological algebra of polyvector fields

$$
\mathrm{HH}^{\bullet}(X)=\bigoplus_{k}\left(\bigoplus_{p+q=k} \mathrm{HH}^{p, q}(X)\right)=\bigoplus_{k}\left(\bigoplus_{p+q=k} H^{q}\left(X, \bigwedge^{p} \Theta_{X}\right)\right)
$$

and then uses Serre duality and $\omega_{X} \cong \mathcal{O}_{X}$ to see how this precisely encodes the Hodge Theory of $X$. More intrinsically, this is the canonical isomorphism

$$
\mathrm{HH}^{\bullet}(X) \cong \mathrm{HH}_{\bullet}(X)[n],
$$

between the Hochschild cohomology and the shifted Hochshild homology of the Calabi-Yau manifold $X$.

Of course the above isomorphism relies heavily on the triviality of the canonical sheaf $\omega_{X} \cong \mathcal{O}_{X}$. In the more general case of $\omega_{X} \cong \mathcal{O}_{X}(m)$ we will consider deformations of the derived category of the punctured affine cone $U_{X}=A_{X} \backslash\{0\}$. Indeed observe that Kontsevich's results still apply since $U_{X}$ is a smooth quasiprojective variety (see [12]). In particular, by using the $\mathbb{C}^{*}$-action which determines the grading, we will focus on the subspace of weight $m$ of the cohomology algebra of polyvector fields on $U_{X}$ and prove the following decomposition result.

Theorem 1.2. Let $X$ be a smooth complex subcanonical projective variety of dimension $n$, and let $m \in \mathbb{Z}$ be the integer such that $\omega_{X} \cong \mathcal{O}_{X}(m)$. Then

$$
\mathrm{HH}^{p, q}\left(U_{X}\right)_{m} \cong H_{\mathrm{prim}}^{n-p+1, q}(X) \oplus H_{\mathrm{prim}}^{n-q, p}(X) .
$$

Theorem 1.2 enables us to reinterpret the (embedded) Hodge Theory of $X$ as a piece of the deformation theory of the derived category of punctured cone $A$. Moreover, we can 
recover Theorem 1.1 as a special case of this result. As a matter of fact if $\operatorname{dim}(X) \geq 2$ and $H^{1}\left(X, \mathcal{O}_{X}(k)\right)=0$ for every $k \in \mathbb{Z}$ then we get an isomorphism

$$
\left(T_{A_{X}}^{1}\right)_{m} \cong H^{1}\left(U_{X}, \Theta_{U_{X}}\right)_{m}=H_{\text {prim }}^{n-1,1}(X)
$$

where we used that the factor $H_{\text {prim }}^{n, 1}(X)$ vanishes by Hard Lefschetz. Similarly, one gets the description for $T_{A_{X}}^{0}$ and $T_{A_{X}}^{2}$.

Acknowledgements. The original idea of using the $T^{1}$ came out after several discussion with Edoardo Sernesi, and we are deeply grateful to him. EF would like to thank his supervisor Miles Reid for constant encouragement and support, and also Stephen Coughlan, Alice Cuzzucoli, Lorenzo De Biase, Paolo Tripoli for useful discussions and suggestions, and Rosemary Taylor for proofreading. CDN and EF would like to thank also Luca Migliorini for his ideas and encouragements in the early stages. Part of this work was completed during the visit of DF and CDN at the University of Warwick for the "2014-2015 Warwick EPSRC Symposium on Derived Categories and Applications", and we would like to thank the organizer of this event. Part of this work was also completed whilst EF was visiting Korea Institute for Advanced Studies (KIAS): the author would like to thank again Professor Miles Reid for inviting and all the staff and academic member of the institute.

\section{The Infinitesimal Deformation Module of an Affine Isolated Singularity}

In this section we will see that - under very mild assumptions - various pieces of the Hodge Theory of a smooth projective variety can be described in terms of the classical deformation modules of the affine cone over it.

2.1. A Quick Review in Deformation Theory. Let $Y$ be a (reasonable) complex scheme and $R$ a local Artin $\mathbb{C}$-algebra; recall that an infinitesimal deformation of $Y$ over $R$ is is the datum of a pull-back diagram

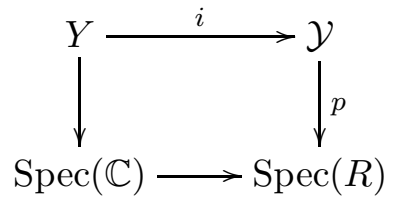

with $i$ a closed immersion and $p$ a flat and proper morphism. Equivalently, a deformation of $Y$ over $R$ can be viewed as a morphism of sheaves of $R$-algebras $\mathcal{O}_{R} \rightarrow \mathcal{O}_{Y}$ such that $\mathcal{O}_{R}$ is flat over $R$ and $\mathcal{O}_{R} \otimes_{R} \mathbb{C} \simeq \mathcal{O}_{Y}$. In more concrete terms, defining a deformation of a scheme $Y$ means defining a flat family of schemes over a fat point whose central fibre is isomorphic to $Y$. Of course there is an obvious notion of isomorphism of deformations, which we will not spell out here in detail.

Studying the Deformation Theory of a scheme $Y$ means indeed analysing the functor

$$
\begin{aligned}
\operatorname{Def}_{Y}: \mathfrak{A n t}_{\mathbb{C}} & \longrightarrow \text { Set } \\
R & \mapsto \frac{\{\text { deformations of } Y \text { over } R\}}{\text { isomorphism }} .
\end{aligned}
$$


which is known to satisfy Schlessinger's axiomatics of deformation functor (for more details see [30]).

It is now well-established that functor $\operatorname{Def}_{Y}$ is governed by a fundamental invariant of the scheme $Y$ - originally envisioned by Grothendieck, then deeply studied by André, Quillen, Deligne, Illusie and many others - that is the cotangent complex $\mathbb{L}_{Y}$. The most concrete way to define the cotangent complex is the following: start with an affine $\mathbb{C}$-scheme $\operatorname{Spec}(B)$ and consider a quasi-free resolution $Q^{\bullet}(B)$. Now apply the Kähler-differential functor to $Q^{\bullet}(B)$ and get the complex of $B$-modules $\Omega_{Q}^{1}{ }^{\bullet}(B)$. Set the (absolute) cotangent complex of $\operatorname{Spec}(B)$ to be

$$
\mathbb{L}_{\mathrm{Spec}(B)}:=\Omega_{Q}^{1} \cdot(B)
$$

and notice that formula (2) provides us with a perfectly well-defined notion in the derived category $D\left(\mathfrak{M o d}_{B}\right)$. As a matter of fact if we change the quasi-free resolution of $B$ we end up with the same object in the derived category. In more formal terms, we can say that the cotangent complex is the total left derived functor (in the sense of Quillen Homotopical Algebra) of the functor of Kähler differentials. The construction of the cotangent complex as given in formula (2) sheafifies, so we can associate to our scheme $Y$ its absolute cotangent complex $\mathbb{L}_{Y}$, which lives in the derived category $D(\mathfrak{Q C o h}(Y))$.

In general the complex $\mathbb{L}_{Y}$ is made of terms lying in both positive and negative degrees and encodes all the invariants determining the functor $\operatorname{Def}_{Y}$ through its Ext groups; we recommend [32] for an introduction to the subject. More precisely we will consider the modules

$$
T_{Y}^{i}:=\operatorname{Ext}_{\mathcal{O}_{Y}}^{i}\left(\mathbb{L}_{Y}, \mathcal{O}_{Y}\right),
$$

which, for $i=0,1,2$ are called the automorphism module, the first-order deformations module, and the obstruction module associated to $Y$, respectively. It is now well-understood that:

(1) $T_{Y}^{0}$ encodes infinitesimal automorphisms of the scheme $Y$;

(2) $T_{Y}^{1}$ parametrises first-order deformations of $Y$, i.e deformations over the ring of dual numbers $\frac{\mathbb{C}[t]}{t^{2}}$

(3) $T_{Y}^{2}$ contains obstructions, meaning that for all surjection $S \rightarrow R$ of local Artin $\mathbb{C}$-algebras the obstructions to lifting a $R$-deformation of $Y$ to a $S$-deformation live in $\operatorname{Ext}^{2}\left(\mathbb{L}_{Y, \mathcal{O}_{Y}}\right)$.

Remark 2.1. The above statements tell us that the cotangent complex $\mathbb{L}_{Y}$ is a much richer object than the deformation functor $\operatorname{Def}_{Y}$ : for example, as a set-valued functor, $\operatorname{Def}_{Y}$ is not able to see the infinitesimal automorphisms of $Y$, which are actually captured by $\mathbb{L}_{Y}$. Also, the higher Ext groups of $\mathbb{L}_{Y}$ describe some more subtle deformations of $Y$ usually called higher obstructions: these objects are extremely important in the new Derived Algebraic Geometry. See, e.g., [33] for the relation between the cohomology of the wedge powers of the (co-)tangent complex of $Y$ and its extended derived deformations. However, in the rest of the paper we will only be interested in studying classical deformation invariants, therefore we will only care about the three above mentioned groups. 
From now on we will focus on the study of the deformation modules attached to an affine cone over a smooth projective variety. Throughout the rest of the paper fix $X$ to be a smooth projectively normal variety and call $A_{X}$ the affine cone over it, so that $A_{X}=\operatorname{Spec}\left(\mathfrak{a}_{X}\right)$, where

$$
\mathfrak{a}_{X}=\bigoplus_{k} H^{0}\left(X, \mathcal{O}_{X}(k)\right) .
$$

We will also suppose everywhere that the projective embedding of $X$ is subcanonical, so that $\omega_{X} \cong \mathcal{O}_{X}(m)$ for some integer $m$. Key references for most of the considerations below are [31, [36]. We stress that their results hold in the more general case of an affine isolated singularity, but here we only consider the case of affine cones over smooth projective varieties.

Consider the $T^{1}$-deformation module

$$
T_{A_{X}}^{1}:=\operatorname{Ext}_{\mathcal{O}_{A_{X}}}^{1}\left(\mathbb{L}_{A_{X}}, \mathcal{O}_{A_{X}}\right),
$$

which measures the first-order deformations of the affine cone $A_{X}$ : in particular notice that it is equipped with a natural grading, induced by the fact that $\mathfrak{a}_{X}$ is a graded algebra itself. The cone $A_{X}$ may deform in several ways and each graded component of $T_{A_{X}}^{1}$ roughly speaking represents the degree of the polynomial we are adding in order to deform. In the case $X$ is a degree $d$ hypersurface with defining polynomial $f$ the graded module $T_{A_{X}}^{1}$ coincides, up to a shift of $-d$, with the Milnor Algebra $\mathcal{M}_{f}$, i.e. we have

$$
T_{A_{X}}^{1}[-d] \cong \mathcal{M}_{f}
$$

as graded modules.

Now, not all deformations of $A_{X}$ lead to another affine cone over a projective variety. The easiest example is the case of $x y=0$ in $\mathbb{C}^{2}$ that is the cone over the points $[1,0],[0,1] \in \mathbb{P}^{1}$. In this case, up to isomorphism the only possible first-order deformation of the cone is given by $x y+\varepsilon=0$, and any element of this family is not a cone over a projective variety except for $\varepsilon=0$. In fact this is straightforward to verify: just notice that the Milnor algebra is

$$
\left(\mathcal{M}_{f}\right)_{m} \cong(\mathbb{C}[x, y] /(x, y))_{m} \cong\left\{\begin{array}{l}
\mathbb{C} \text { if } m=0 \\
0 \text { if } m \neq 0
\end{array},\right.
$$

hence $\left(T_{A_{X}}^{1}\right)_{0}=\left(\mathcal{M}_{f}\right)_{2}=0$. A very natural question arises: what are the deformations of $A_{X}$ that lead to family of affine cones over a projective variety? The naive answer is that the polynomials that we add in order to deform must be homogeneous of the same degree as the (homogeneous) equations of $A_{X}$. Luckily, this is also the correct one.

So far, all of what we said is very classical: under the above interpretation, the degree 0 piece of the deformation module of the affine cone over the projective variety $X$ represents the embedded first-order deformations of $X$ inside $\mathbb{P}^{N}$. Somehow more precisely, we have an exact sequence

$$
\ldots \rightarrow H^{1}\left(X, \mathcal{O}_{X}\right) \rightarrow\left(T_{A_{X}}^{1}\right)_{0} \rightarrow H^{1}\left(X, \Theta_{X}\right) \rightarrow H^{2}\left(X, \mathcal{O}_{X}\right) \rightarrow \ldots
$$


Notice that if the two side terms are both zero, we have an isomorphism between $\left(T_{A_{X}}^{1}\right)_{0}$ and $H^{1}\left(X, \Theta_{X}\right)$, which allows us to identify $\left(T_{A_{X}}^{1}\right)_{0}$ with all infinitesimal deformations of $X$. This is for example the case of a smooth Calabi-Yau of dimension $\geq 3$; by definition we have $H^{1}\left(X, \mathcal{O}_{X}\right)=H^{2}\left(X, \mathcal{O}_{X}\right)=0$. This coincides with the standard fact that all Calabi-Yau from dimension 3 onwards are projective, while for example in the K3 case we have a 19-dimensional algebraic family inside a 20 -dimensional deformation space, recorded by the fact $H^{2}\left(X, \mathcal{O}_{X}\right) \cong H^{0}\left(X, \omega_{X}\right) \cong \mathbb{C}$. In general when either $H^{0,1}(X)$ or $H^{0,2}(X)$ are non zero there is generally a difference between embedded deformations and non-embedded ones.

2.2. $T^{1}$ and Hodge Theory. Now we want to explore deeper the relation between $T_{A_{X}}^{1}$, the smooth projective variety 11 , and the punctured cone $U_{X}:=A_{X} \backslash\{0\}$.

Lemma 2.2. For every $k \in \mathbb{Z}$, the relative tangent sheaf exact sequence

$$
0 \rightarrow \Theta_{U_{X} / X} \rightarrow \Theta_{U_{X}} \stackrel{\mathrm{d} \pi}{\rightarrow} \pi^{*}\left(\Theta_{X}\right) \rightarrow 0
$$

induces a long exact sequence

$$
\ldots \rightarrow H^{1}\left(X, \mathcal{O}_{X}(k)\right) \rightarrow H^{1}\left(U_{X}, \Theta_{U_{X}}\right)_{k} \rightarrow H^{1}\left(X, \Theta_{X}(k)\right) \stackrel{\lambda}{\rightarrow} H^{2}\left(X, \mathcal{O}_{X}(k)\right) \rightarrow \ldots
$$

where the maps $\lambda$ are the Lefschetz operators.

Proof. The Euler vector field gives a trivialization $\Theta_{U_{X} / X} \cong \mathcal{O}_{U_{X}}$, see [11]. We therefore get the short exact sequence

$$
0 \rightarrow \mathcal{O}_{U_{X}} \rightarrow \Theta_{U_{X}} \rightarrow \pi^{*}\left(\Theta_{X}\right) \rightarrow 0
$$

and so, passing to cohomology, the long exact sequence

$$
\ldots \rightarrow \bigoplus_{k \in \mathbb{Z}} H^{1}\left(X, \mathcal{O}_{X}(k)\right) \stackrel{\lambda}{\rightarrow} \bigoplus_{k \in \mathbb{Z}} H^{1}\left(U_{X}, \Theta_{U_{X}}\right)_{k} \rightarrow \bigoplus_{k \in \mathbb{Z}} H^{1}\left(X, \Theta_{X}(k)\right) \rightarrow \bigoplus_{k \in \mathbb{Z}} H^{2}\left(X, \mathcal{O}_{X}(k)\right) \rightarrow \ldots
$$

where the grading on $H^{1}\left(U_{X}, \Theta_{U_{X}}\right)$ is induced by the $\mathbb{C}^{*}$-action, and the connecting homomorphism $\lambda$ is the cup product with the extension class

$$
\Lambda:=\left[0 \rightarrow \mathcal{O}_{U_{X}} \rightarrow \Theta_{U_{X}} \rightarrow \pi^{*} \Theta_{X} \rightarrow 0\right],
$$

which is an element in

$$
\operatorname{Ext}_{U_{X}}^{1}\left(\pi^{*} \Theta_{X}, \mathcal{O}_{U_{X}}\right) \cong H^{1}\left(U_{X}, \pi^{*} \Omega_{X}^{1}\right) \cong \bigoplus_{s} H^{1}\left(X, \Omega_{X}^{1}(s)\right),
$$

see [31] ([Lemma 1, page 158]) and [34]. Notice that the map $\lambda$ is not a priori a morphism of graded modules: we should expect it to have several homogeneous components

$$
\lambda_{s}: H^{i}\left(X, \Theta_{X}(k)\right) \longrightarrow H^{i+1}\left(X, \mathcal{O}_{X}(k+s)\right),
$$

which are identified with cohomology classes in $H^{1}\left(X, \Omega^{1}(s)\right)$. So our next step consists in showing that actually $\lambda$ reduces to its degree zero component $\lambda_{0}$, i.e., that $\Lambda$ consists into

\footnotetext{
${ }^{1}$ Actually, everything in what follows holds more generally for quasi-smooth varieties in weighted projective spaces.
} 
a single cohomology class in $H^{1}\left(X, \Omega^{1}\right)$. To see this, recall from [2] (see [25] for a more modern treatment) that given a line bundle $L$ on a smooth complex manifold $X$, if we denote by $L^{\circ}$ the total space of the dual bundle $L^{*}$ with the zero section removed, then we have a canonical short exact sequence of sheaves of $\mathcal{O}_{L^{\circ}}$-modules

$$
0 \rightarrow \pi^{*} \Omega_{X}^{1} \rightarrow \Omega_{L^{\circ}}^{1} \rightarrow \mathcal{O}_{L^{\circ}} \rightarrow 0 .
$$

Pushing forward to $X$ we get for every $k \in \mathbb{Z}$ a short exact sequence

$$
0 \rightarrow \Omega_{X}^{1}(k) \rightarrow \mathcal{L}_{k} \rightarrow L^{\otimes k} \rightarrow 0,
$$

where $\mathcal{L}_{k}$ denotes the degree $k$ component of $\pi_{*} \Omega_{L^{\circ}}^{1}$. In particular, if $L=\mathcal{O}_{X}(1)$, so that $L^{\circ} \cong U_{X}$, we get the short exact sequences

$$
0 \rightarrow \Omega_{X}^{1}(k) \rightarrow\left(\pi_{*} \Omega_{U_{X}}^{1}\right)_{k} \rightarrow \mathcal{O}_{X}(k) \rightarrow 0,
$$

and the projection formula together with the isomorphisms $\pi^{*} \mathcal{O}_{X} \cong \mathcal{O}_{U_{X}} \cong \pi^{*} \mathcal{O}_{X}(k)$, shows that these are indeed all obtained from the single short exact sequence

$$
0 \rightarrow \Omega_{X}^{1} \rightarrow\left(\pi_{*} \Omega_{U_{X}}^{1}\right)_{0} \rightarrow \mathcal{O}_{X} \rightarrow 0
$$

by tensoring it by $\mathcal{O}_{X}(k)$. This implies that the extension class $\left[0 \rightarrow \Omega_{X}^{1}(k) \rightarrow\left(\pi_{*} \Omega_{U_{X}}^{1}\right)_{k} \rightarrow\right.$ $\left.\mathcal{O}_{X}(k) \rightarrow 0\right]$ is actually independent of $k$, and so the total extension class $\left[0 \rightarrow \pi^{*} \Omega_{X}^{1} \rightarrow\right.$ $\left.\Omega_{U_{X}}^{1} \rightarrow \mathcal{O}_{U_{X}} \rightarrow 0\right]$ reduces to the extension class $\left[0 \rightarrow \Omega_{X}^{1} \rightarrow\left(\pi_{*} \Omega_{U_{X}}^{1}\right)_{0} \rightarrow \mathcal{O}_{X} \rightarrow 0\right]$, which is an element in $\operatorname{Ext}_{X}^{1}\left(\Theta_{X}, \mathcal{O}_{X}\right) \cong H^{1}\left(X, \Omega_{X}^{1}\right)$, see [8]. Since the short exact sequence $0 \rightarrow \pi^{*} \Omega_{X}^{1} \rightarrow \Omega_{U_{X}}^{1} \rightarrow \mathcal{O}_{U_{X}} \rightarrow 0$ is the dual of the short exact sequence $0 \rightarrow \mathcal{O}_{U_{X}} \rightarrow$ $\Theta_{U_{X}} \rightarrow \pi^{*}\left(\Theta_{X}\right) \rightarrow 0$ we finally see that the connecting homomorphism $\lambda$ is indeed of degree zero and is given by the cup product with a distinguished element $\Lambda$ in $H^{1}\left(X, \Omega_{X}^{1}\right)$. Since $\lambda$ is a degree zero operator, it preserves the gradings, and so for every degree $k$ we have a long exact sequence

$$
\ldots \rightarrow H^{1}\left(X, \mathcal{O}_{X}(k)\right) \stackrel{\lambda}{\rightarrow} H^{1}\left(U_{X}, \Theta_{U_{X}}\right)_{k} \rightarrow H^{1}\left(X, \Theta_{X}(k)\right) \rightarrow H^{2}\left(X, \mathcal{O}_{X}(k)\right) \rightarrow \ldots
$$

To conclude we have to identify $\Lambda$ with the class of an hyperplane. Again, we refer to [2], where it is shown that, for a general line bundle $L$, the extension class $\left[0 \rightarrow \Omega_{X}^{1} \rightarrow \mathcal{L}_{0} \rightarrow\right.$ $\left.\mathcal{O}_{X} \rightarrow 0\right]$ is the first Chern class $c_{1}(L)$. So, for $L=\mathcal{O}_{X}(1)$ we find that the extension class is $c_{1}\left(\mathcal{O}_{X}(1)\right)$, as desired.

Corollary 2.3. Let $X$ be a smooth subcanonical projectively normal variety of dimension $n$, and let $m \in \mathbb{Z}$ be the integer such that $\omega_{X} \cong \mathcal{O}_{X}(m)$. Then the relative tangent sheaf exact sequence

$$
0 \rightarrow \Theta_{U_{X} / X} \rightarrow \Theta_{U_{X}} \stackrel{\mathrm{d} \pi}{\rightarrow} \pi^{*}\left(\Theta_{X}\right) \rightarrow 0
$$

induces a long exact sequence

$$
\ldots \rightarrow H^{n-1,0}(X) \stackrel{\lambda}{\rightarrow} H^{n, 1}(X) \rightarrow H^{1}\left(U_{X}, \Theta_{U_{X}}\right)_{m} \rightarrow H^{n-1,1}(X) \stackrel{\lambda}{\rightarrow} H^{n, 2}(X) \rightarrow \ldots
$$

where the maps $\lambda$ are the Lefschetz operators. 
Proof. Since $\omega_{X} \cong \mathcal{O}_{X}(m)$, Serre duality gives canonical isomorphisms

$$
H^{i}\left(X, \Theta_{X}(m)\right) \simeq H^{n-1, i}(X)
$$

and

$$
H^{i}\left(X, \mathcal{O}_{X}(m)\right) \simeq H^{n, i}(X) .
$$

The result then follows from Lemma 2.2 for $k=m$.

We are now ready to prove the main result of this section.

Theorem 2.4. Let $X$ be a smooth subcanonical projectively normal variety of dimension $n$, and let $m \in \mathbb{Z}$ be the integer such that $\omega_{X} \cong \mathcal{O}_{X}(m)$. There is a natural isomorphism

$$
H^{1}\left(U, \Theta_{U_{X}}\right)_{m} \cong H_{\text {prim }}^{n-1,1}(X) .
$$

Proof. Consider the long exact sequence

$$
\ldots \rightarrow H^{n-1,0}(X) \stackrel{\lambda_{n-1,0}}{\rightarrow} H^{n, 1}(X) \rightarrow H^{1}\left(U_{X}, \Theta_{U_{X}}\right)_{m} \rightarrow H^{n-1,1}(X) \stackrel{\lambda_{n-1,1}}{\rightarrow} H^{n, 2}(X) \rightarrow \ldots
$$

from Corollary 2.3, It induces the short exact sequence

$$
0 \rightarrow \operatorname{coKer}\left(\lambda_{n-1,0}\right) \rightarrow H^{1}\left(U_{X}, \Theta_{U_{X}}\right)_{m} \rightarrow \operatorname{Ker}\left(\lambda_{n-1,1}\right) \rightarrow 0 .
$$

Now notice that, by definition, $\operatorname{Ker}\left(\lambda_{n-1,1}\right)=H^{n-1,1}(X)_{\text {prim }}$, while $\operatorname{coKer}\left(\lambda_{n-1,0}\right)$ is zero since, $\lambda_{n-1,0}$ is an isomorphism by Hard Lefschetz.

What we have to do now is connect the previous result to the $T_{A_{X}}^{1}$, the module of first-order deformations of the affine cone of $X$.

Theorem 2.5. Let $X$ be a smooth subcanonical projectively normal variety of dimension $n>1$, and let $m \in \mathbb{Z}$ be the integer such that $\omega_{X} \cong \mathcal{O}_{X}(m)$. If $H^{1}\left(X, \mathcal{O}_{X}(k)\right)=0$ for every $k \in \mathbb{Z}$, then we have

$$
\left(T_{A_{X}}^{1}\right)_{m} \cong H_{\text {prim }}^{n-1,1}(X)
$$

Proof. From [31] we have that $T_{A_{X}}^{1}$ fits into the exact sequence

$$
0 \rightarrow T_{A_{X}}^{1} \rightarrow H^{1}\left(U_{X}, \Theta_{U_{X}}\right) \rightarrow H^{1}\left(U_{X},\left.\left(\Theta_{\mathbb{C}^{N+1}}\right)\right|_{U_{X}}\right) \cong H^{1}\left(U_{X}, \mathcal{O}_{U_{X}}\right)^{N+1}
$$

Since $H^{1}\left(U_{X}, \mathcal{O}_{U_{X}}\right) \cong \bigoplus_{k} H^{1}\left(X, \mathcal{O}_{X}(k)\right)$, we see that if $H^{1}\left(X, \mathcal{O}_{X}(k)\right)=0$ for every $k \in \mathbb{Z}$, then $T_{A_{X}}^{1} \cong H^{1}\left(U_{X}, \Theta_{U_{X}}\right)$. The conclusion the follows from Theorem 2.4.

Corollary 2.6. Under the same hypothesis of the theorem above, we have that in general the degree $k$ component of the $T_{A_{X}}^{1}$ is given by

$$
\left(T_{A_{X}}^{1}\right)_{k} \cong \operatorname{Ker}\left(\lambda: H^{1}\left(X, \Omega^{n-1}(k-m)\right) \rightarrow H^{2}\left(X, \omega_{X}(k-m)\right) .\right.
$$

Remark 2.7. What kind of varieties satisfies the condition $H^{1}\left(X, \mathcal{O}_{X}(k)\right)=0$ for every $k \in \mathbb{Z}$ that appears in Theorem 2.5 above? By Kodaira vanishing one sees that all smooth Fano manifolds and simply connected projective Calabi-Yau manifolds satisfy this condition. Also, every arithmetically Cohen-Macaulay projective variety (and so, in particular projective spaces and their products, projective complete intersections, Grassmann 
manifolds and Schubert subvarieties, flag manifolds and generalized flag manifolds) of dimension at least 2 satisfies it. Notice that, if $\operatorname{dim} X \geq 2$, the vanishing condition condition $H^{1}\left(X, \mathcal{O}_{X}(k)\right)=0$ for every $k \in \mathbb{Z}$ is actually equivalent to the condition $\operatorname{depth}_{0} A_{X} \geq 3$. Namely, we can identify $H^{1}\left(U_{X}, \mathcal{O}_{U_{X}}\right)$ with $H_{\mathfrak{m}}^{2}\left(A_{X}, \mathcal{O}_{A_{X}}\right)$, the second local cohomology group of $A_{X}$ at the maximal (irrelevant) ideal, and the vanishing of this is by definition the same request as $\operatorname{depth}_{0} A_{X} \geq 3$.

For $\operatorname{dim} X \geq 2$ the simplest example of projective manifolds for which $H^{1}\left(X, \mathcal{O}_{X}(k)\right)$ does not vanish for every $k$ given by Abelian varieties: for them, theorem 2.4 still holds, but the problem of determining the image of $T_{A}^{1}$ inside $H^{1}\left(U_{X}, \Theta_{U_{X}}\right)$ remains open.

2.3. Obstructions and Automorphisms. Now we look at the Obstruction Theory of the cone $A_{X}$. Infinitesimal obstructions to deformations of $A_{X}$ live inside

$$
T_{A_{X}}^{2}:=\operatorname{Ext}_{\mathcal{O}_{A_{X}}}^{2}\left(\Omega_{A_{X}}^{1}, \mathcal{O}_{A_{X}}\right) .
$$

Let us stick to the case of $\operatorname{depth}_{0} A_{X} \geq 3$ and $\operatorname{dim} X \geq 2$, so that $H^{1}\left(X, \mathcal{O}_{X}(k)\right)=0$ for any $k$ as in the previous section. Following [31], we can identify $T_{A_{X}}^{2}$ with $H^{1}\left(U_{X}, N_{U_{X}}\right)$, where $N_{U_{X}}$ is the normal bundle of $U_{X}$ in $\mathbb{C}^{N+1}$. From the defining exact sequence

$$
\left.0 \rightarrow \Theta_{U_{X}} \rightarrow \Theta_{\mathbb{C}^{N+1}}\right|_{U_{X}} \rightarrow N_{U_{X}} \rightarrow 0
$$

for $N_{U_{X}}$ we obtain the long exact sequence

$$
\ldots \rightarrow 0 \rightarrow H^{1}\left(U_{X}, N_{U_{X}}\right) \rightarrow H^{2}\left(U_{X}, \Theta_{U_{X}}\right) \rightarrow\left(\bigoplus_{k} H^{2}\left(X, \mathcal{O}_{X}(k)\right)\right)^{N+1} \rightarrow \ldots
$$

in cohomology, so that if $H^{2}\left(X, \mathcal{O}_{X}(k)\right)=0$ for any $k$ we have an isomorphism

$$
T_{A_{X}}^{2} \cong H^{2}\left(U_{X}, \Theta_{U_{X}}\right) \text {. }
$$

Notice that the condition $H^{i}\left(X, \mathcal{O}_{X}(k)\right)=0$ for any $k$ in $\mathbb{Z}$ and for $i=1,2$ is in particular satisfied by every arithmetically Cohen-Macaulay variety of dimension at least 3 . From Lemma 2.2 we have the exact sequences

$$
\ldots \rightarrow 0 \rightarrow\left(T_{A_{X}}^{2}\right)_{k} \rightarrow H^{2}\left(X, \Theta_{X}(k)\right) \stackrel{\lambda}{\rightarrow} H^{3}\left(X, \mathcal{O}_{X}(k)\right) \rightarrow \ldots
$$

where the maps $\lambda$ are the Lefschetz operators. Let us restrict to the cases $k=0$ and $k=m$, where $m$ is the integer such that $\omega_{X} \cong \mathcal{O}(m)$. For $k=0$ we find the exact sequence

$$
\ldots \rightarrow 0 \rightarrow\left(T_{A_{X}}^{2}\right)_{0} \rightarrow H^{2}\left(X, \Theta_{X}\right) \stackrel{\lambda}{\rightarrow} H^{3}\left(X, \mathcal{O}_{X}\right) \rightarrow \ldots
$$

which identifies $\left(T_{A}^{2}\right)_{0}$ with a subspace of $H^{2}\left(X, \Theta_{X}\right)$, which is the space containing the obstruction to (non-immersed) deformations of $X$. If moreover $\lambda: H^{2}\left(X, \Theta_{X}\right) \rightarrow H^{3}\left(X, \mathcal{O}_{X}\right)$ is the zero map (as is the case, e.g., if $H^{3}\left(X, \mathcal{O}_{X}\right)$ vanishes), then we have an isomorphism $\left(T_{A}^{2}\right)_{0} \cong H^{2}\left(X, \Theta_{X}\right)$. If instead we look at the $k=m$ case, then by Corollary 2.3 we have the long exact sequence

$$
\ldots \rightarrow H^{n-1,1}(X) \stackrel{\lambda}{\rightarrow} H^{n, 2}(X) \rightarrow\left(T_{A}^{2}\right)_{m} \rightarrow H^{n-1,2}(X) \stackrel{\lambda}{\rightarrow} H^{n, 3}(X) \rightarrow \ldots
$$

and so we get the following 
Theorem 2.8. Let $X$ be a smooth subcanonical projectively normal variety of dimension $n$, and let $m \in \mathbb{Z}$ be the integer such that $\omega_{X} \cong \mathcal{O}_{X}(m)$. If $H^{i}\left(X, \mathcal{O}_{X}(k)\right)=0$ for every $k \in \mathbb{Z}$, and for $i=1,2$ then we have a natural isomorphism

$$
\left(T_{A}^{2}\right)_{m} \cong H_{\text {prim }}^{n-2,1}(X) \text {. }
$$

Proof. By the above discussion, we have a natural short exact sequence

$$
0 \rightarrow \operatorname{coKer}\left(\lambda_{n-1,1}\right) \rightarrow\left(T_{A}^{2}\right)_{m} \rightarrow \operatorname{Ker}\left(\lambda_{n-1,2}\right) \rightarrow 0 .
$$

By Hard Lefschetz, $\operatorname{coKer}\left(\lambda_{n-1,1}\right)=0$, and

$$
\operatorname{Ker}\left(\lambda_{n-1,2}\right)=\lambda_{n-2,1} \operatorname{Ker}\left(\lambda_{n-1,2} \lambda_{n-2,1}\right) \cong H_{\text {prim }}^{n-2,1}(X)
$$

A similar result holds for the $T_{A_{X}}^{0}$, the module that parametrize the infinitesimal automorphism of the affine cone $A_{X}$. If $\operatorname{depth}_{0} A_{X} \geq 2$ (which is satisfied, e.g., if $X$ is normal), we have $T_{A_{X}}^{0} \cong H^{0}\left(U_{X}, \Theta_{U_{X}}\right)$ and so Corollary 2.3 gives the long exact sequence

$$
0 \rightarrow H^{n, 0}(X) \rightarrow\left(T_{A}^{0}\right)_{m} \rightarrow H^{n-1,0}(X) \stackrel{\lambda_{n-1,0}}{\rightarrow} H^{n, 1}(X) \rightarrow \ldots
$$

and so the short exact one

$$
0 \rightarrow H^{n, 0}(X) \rightarrow\left(T_{A}^{0}\right)_{m} \rightarrow \operatorname{coKer}\left(\lambda_{n-1,0}\right) \rightarrow 0 .
$$

By Hard Lefschetz, $\lambda_{n-1,0}$ is an isomorphism and so $\operatorname{coKer}\left(\lambda_{n-1,0}\right)=0$, while

$$
H^{n, 0}(X)=H_{\text {prim }}^{n, 0}(X) \text {. }
$$

Thus we have

Theorem 2.9. Let $X$ of dimension $n$ be smooth, projective, with $\omega_{X} \cong \mathcal{O}_{X}(m)$. Then we have an isomorphism

$$
\left(T_{A}^{0}\right)_{m} \cong H_{\text {prim }}^{n, 0}(X)
$$

2.4. A SINGULAR appendix: how to compute Hodge numbers using the $T^{i}$. One of the many applications of our theorems on the $T_{A_{X}}^{1}$ and the $T_{A_{X}}^{2}$ is a concrete tool to compute part of the Hodge structure of a smooth projective variety. We recall from the previous section that, under appropriate hypothesis on depth at the vertex, we can identify

$$
\begin{aligned}
\left(T_{A_{X}}^{1}\right)_{m} & \cong H_{\mathrm{prim}}^{n-1,1}(X) \\
\left(T_{A_{X}}^{2}\right)_{m} & \cong H_{\text {prim }}^{n-2,1}(X),
\end{aligned}
$$

where as usual $\omega_{X} \cong \mathcal{O}_{X}(m)$. The key is that both $T^{1}$ and $T^{2}$ are easily computable, especially using computer algebra languages such as SINGULAR (see [16]), already endowed with efficient built-in tools. Suppose we start from $X \subset \mathbb{P}^{N}$ a smooth projective variety, with $X=V(I)$, where $I=\left(f_{1}, \ldots, f_{m}\right)$. Then the instruction

module $\mathrm{T}_{-} 1=\mathrm{T}_{-} 1(\mathrm{I})$;

module T_2=T_2(I);

hilb $\left(T_{-} 1,2\right)$;

hilb (T_2,2); 
computes the dimension of both $T_{A_{X}}^{1}[-d]$ and $T_{A_{X}}^{2}[-d]$, where $d=\max \left\{\operatorname{deg}\left(f_{i}\right)\right\}$ and then lists the dimensions of the various graded components. Let us pursue a couple of nontrivial example in detail:

2.4.1. A (Gushel-Mukai) Fano Threefold of Degree 10 and Coindex 1. Consider the case of $X=G r(2,5) \cap H_{1} \cap H_{2} \cap Q$ a threefold complete intersection in the Grassmannian of 2-planes in $\mathbb{C}^{5}$ given by two hyperplane sections and one quadric, considered for example in [29] and [15] for its connections with HyperKähler geometry. Since the canonical class of the Grassmannian is $\omega_{G r(2,5)} \cong \mathcal{O}_{G r(2,5)}(-5)$, by adjunction $X$ is a Fano of index 1, that is $\omega_{X}=\mathcal{O}_{X}(-1)$. By Kodaira vanishing we have $h^{i, 0}(X)=0$ for $i=1,2,3$, and by Lefschetz hyperplane theorem we have $H^{1,1}(X)=H^{2,2}(X) \cong \mathbb{C}$. Thus the only Hodge piece missing is $H^{2,1}(X)=H_{\text {prim }}^{2,1}(X)$, and by our theorem we have

$$
H_{\text {prim }}^{2,1}(X) \cong\left(T_{A_{X}}^{1}\right)_{-1}
$$

Let us produce a code in SINGULAR:

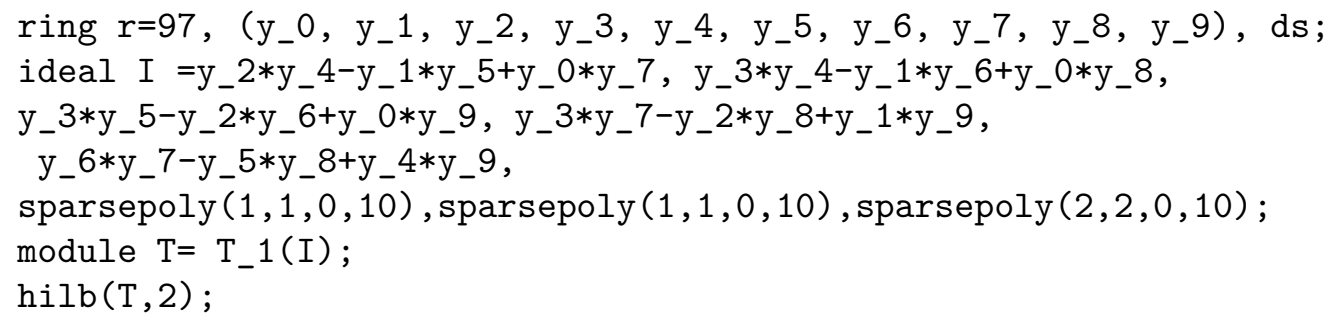

the first 5 equations in the ideal are nothing but the Plücker relations of $G r(2,5)$ embedded in $\mathbb{P}^{9}$, with three generic hyperplane sections and one quadratic equation (actually SINGULAR can check if those four equations form a regular sequence). The dimension of the graded component we get in return is

$1,10,22,11,1$

where we have to look at the component of degree 1 , since $\left(T_{A_{X}}^{1}\right)_{-1}=\left(T_{A_{X}}^{1}\right)[-2]_{1}$, and this is 10. If needed, we can ask for an explicit monomial basis for $H^{2,1}(X)$, using the command

kbase $(\mathrm{T}, 1)$;

Note that $\left(T_{A_{X}}^{1}\right)_{0}$ is 22-dimensional: this agrees (and the same for the Hodge numbers) with the computation considered in [15]. In particular its lower Hodge diamond is

$\begin{array}{ccccccc}0 & & 10 & & 10 & & 0 \\ & 0 & & 1 & & 0 & \\ & & 0 & & 0 & & \end{array}$

2.4.2. A Pfaffian-Calabi Yau Threefold. Consider now the case of a Pfaffian-Calabi Yau Threefold, as in the work of [4] and [26]. We define

$$
P=G \cap Q_{1} \cap Q_{2} \cap H,
$$


where $G=\operatorname{Gr}(2,5)$ as before, $Q_{1}$ and $Q_{2}$ are quadrics, $H$ is an hyperplane. By a computation analogous to the previous example it is easy to see that $P$ is a Calabi-Yau threefold: the dimension of the graded component of the $T^{1}$ of its affine cone are

$2,19,61,101,82,29,3$

In particular we find the lower Hodge diamond

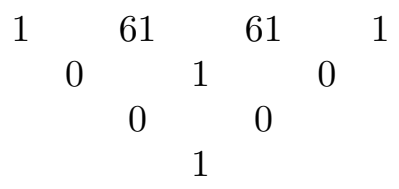

2.4.3. A weighted example. As said before, the method we implemented works well also in the weighted projective space case. As an example, we look at the online database [9] where several thousands of families of quasi-smooth Fano threefolds are listed. In SINGULAR, we can deal with weighted projective space by specifying a weighted order on the monomial, namely using the command

wp $\left(\mathrm{a}_{-} 0, \ldots, \mathrm{a}_{-} \mathrm{n}\right)$

where the $a_{i}$ are the chosen weights. As an example, we pick $X_{6,7} \subset \mathbb{P}(1,1,2,2,3,5)$, corresponding to the entry 5839 in the database [9]. This is a codimension 2 Fano threefold of index 1 , degree $7 / 10$ and with a $3 \times \frac{1}{2}(1,1,1), \frac{1}{5}(1,2,3)$ as Basket. Computing the $T^{1}$ in the same exact way as before we get $\left(T_{A}^{1}\right)_{-1}=39$, and we can then draw the lower Hodge diamond as

$\begin{array}{lllllll}0 & & 39 & & 39 & & 0 \\ & 0 & & 1 & & 0 & \\ & & 0 & & 0 & & \\ & & & 1 & & & \end{array}$

\section{Deformations of Derived Categories and Hodge Theory}

3.1. A Primer on Noncommutative Schemes and Hochschild Structures. Remark 2.1 suggests that one natural way to generalise the notion of deformation of a $\mathbb{C}$-scheme $Y$ is by considering derived deformations. Namely, derived deformations of $Y$ are already encoded in the cotangent complex. On the other hand another interesting generalisation consists in deforming $Y$ as a noncommutative schemes - whatever these structures could be.

The theory of noncommutative schemes, usually known as Noncommutative Algebraic Geometry, is very much a developing subject, whose fundamentals have not been completely settled yet; however the basic idea - which dates back to Grothendieck and has recently gone through a rapid development - consists of observing that the geometry of $Y$ does not really depend on the scheme as a space, but rather on the derived category $D(\mathfrak{C o h}(Y))$ or better on its dg-enhancements or, more generally $A_{\infty}$-enanchments. Therefore, a (nonnecessarily commutative) scheme over $\mathbb{C}$ can be thought as the datum of an $A_{\infty}$-category over $\mathbb{C}$ : those which are quasi-equivalent to a dg-category of coherent sheaves over a classical scheme will encode the usual commutative schemes, whereas the others will be called 
noncommutative schemes. In particular, a $A_{\infty}$ - deformation of the commutative scheme $Y$ will be a deformation of the dg-category category $D(\mathfrak{C o h}(Y))$ as an $A_{\infty}$-category [6]. Exactly as the infinitesimal deformation theory of an associative algebra (or more generally an $A_{\infty}$-algebra) is governed by its Hochschild cohomology, so happens for the infinitesimal deformation theory of $A_{\infty}$-categories. In particular, the $A_{\infty}$-deformations of a commutative $\mathbb{C}$-scheme $Y$ are governed by

$$
\mathrm{HH}^{\bullet}(Y):=\mathrm{HH}^{\bullet}(D \mathfrak{C o h}(Y)) \cong \operatorname{Ext}_{Y \times Y}^{\bullet}\left(\mathcal{O}_{\Delta}, \mathcal{O}_{\Delta}\right)
$$

where $\mathcal{O}_{\Delta}$ stands for the structure sheaf of the diagonal in $Y \times Y$, see [12].

There are some even more concrete interpretations of Hochschild cohomology: as a matter of fact if $Y$ is a smooth quasiprojective variety the Hochschild-Kostant-Rosenberg Theorem (see [12]) establishes an isomorphism between $\mathrm{HH}^{\bullet}(Y)$ and the (cohomology) algebra of polyvector fields on $Y$, namely

$$
\mathrm{HH}^{\bullet}(Y) \cong \bigoplus_{p, q} H^{q}\left(Y, \bigwedge^{p} \Theta_{Y}\right)
$$

Notice that the algebra of polyvector fields $\bigoplus_{p, q} H^{q}\left(Y, \wedge^{p} \Theta_{Y}\right)$ is known to be the tangent space at $Y$ to the extended moduli supermanifold of complex structures (see [3]) and is also related to the derived moduli of non-commutative polarized schemes recently studied by Behrend and Noohi [5]. Finally, if $Y$ is a projective Calabi-Yau, Serre duality gives a canonical isomorphism

$$
\mathrm{HH}^{\bullet}(Y) \cong \mathrm{HH}_{\bullet}(Y)[n]
$$

where the Hochschild homology of $Y$ is identified the "vertical slices" of the Hodge diamond of $Y$ :

$$
\mathrm{HH}_{\bullet}(Y)=\bigoplus_{k} \mathrm{HH}_{k}(Y)=\bigoplus_{k}\left(\bigoplus_{p-q=k} H^{p, q}(Y)\right)
$$

3.2. Hochschild Cohomology of Punctured Affine Cones. In the context of Hochschild structures, the results in Section 2.2 and Section 2.3 have beautiful generalizations. We start proving the following straightforward generalization of Lemma 2.2 .

Lemma 3.1. For every $k \in \mathbb{Z}$, and for every $p \geq 0$, the relative tangent sheaf exact sequence

$$
0 \rightarrow \Theta_{U_{X} / X} \rightarrow \Theta_{U_{X}} \stackrel{\mathrm{d} \pi}{\rightarrow} \pi^{*}\left(\Theta_{X}\right) \rightarrow 0
$$

induces a long exact sequence

$$
\ldots \rightarrow H^{q}\left(X, \bigwedge^{p-1} \Theta_{X}(k)\right) \rightarrow H^{q}\left(U_{X}, \bigwedge^{p} \Theta_{U_{X}}\right)_{k} \rightarrow H^{q}\left(X, \bigwedge^{p} \Theta_{X}(k)\right) \stackrel{\lambda}{\rightarrow} H^{q+1}\left(X, \stackrel{p-1}{\bigwedge} \Theta_{X}(k)\right) \rightarrow \ldots
$$

where the maps $\lambda$ are the contractions with the hyperplane class in $H^{1}\left(X, \Omega_{X}^{1}\right)$, and where for $p=0$ one is setting $\bigwedge^{-1} \Theta_{X}=0$. 
Proof. The differential $\mathrm{d} \pi: \Theta_{U_{X}} \rightarrow \pi^{*}\left(\Theta_{X}\right)$ induces a short exact sequence of sheaves of $\mathcal{O}_{U_{X}}$-algebras

$$
0 \rightarrow \operatorname{ker}(\mathrm{d} \pi) \rightarrow \grave{\bigwedge} \Theta_{U_{X}} \stackrel{\mathrm{d} \pi}{\rightarrow} \pi^{*} \grave{\Lambda} \Theta_{X} \rightarrow 0
$$

which in every homogeneous degree $p$ reads

$$
0 \rightarrow \pi^{*} \bigwedge^{p-1} \Theta_{X} \rightarrow \bigwedge^{p} \Theta_{U_{X}} \rightarrow \pi^{*} \bigwedge^{p} \Theta_{X} \rightarrow 0
$$

since the leftmost term in the relative tangent sheaf exact sequence is a trivial line bundle (see, e.g., [23, Theorem 4.1.3]). Since (41) is a square zero extension, the connecting homomorphisms in the long exact sequence

$\ldots \rightarrow H^{q}\left(U_{X}, \pi^{*} \stackrel{\bullet-1}{\bigwedge} \Theta_{X}\right) \rightarrow H^{q}\left(U_{X}, \grave{\bigwedge} \Theta_{U_{X}}\right) \rightarrow H^{q}\left(U_{X}, \pi^{*} \stackrel{\bigwedge^{\prime}}{\Theta_{X}}\right) \stackrel{\lambda}{\rightarrow} H^{q+1}\left(U_{X}, \pi^{*} \stackrel{\bullet-1}{\bigwedge} \Theta_{X}\right) \rightarrow \ldots$

are given by the connecting homomorphism for the degree 1 sequence

$$
\ldots \rightarrow H^{q}\left(U_{X}, \mathcal{O}_{U_{X}}\right) \rightarrow H^{q}\left(U_{X}, \Theta_{U_{X}}\right) \rightarrow H^{q}\left(U_{X}, \pi^{*} \Theta_{X}\right) \stackrel{\lambda}{\rightarrow} H^{q+1}\left(U_{X}, \mathcal{O}_{U_{X}}\right) \rightarrow \ldots
$$

extended as a (graded) derivation. By Lemma 2.2 we know that this is given by the contraction with the hyperplane class seen as a degree zero element in $H^{1}\left(U_{X}, \pi^{*} \Omega_{X}^{1}\right)$. In particular, $\lambda$ will be degree preserving, and so we get, for every $p$ and every $k$ the long exact sequence

$\ldots \rightarrow H^{q}\left(X, \bigwedge^{p-1} \Theta_{X}(k)\right) \rightarrow H^{q}\left(U_{X}, \bigwedge^{p} \Theta_{U_{X}}\right)_{k} \rightarrow H^{q}\left(X, \bigwedge^{p} \Theta_{X}(k)\right) \stackrel{\lambda}{\rightarrow} H^{q+1}\left(X, \bigwedge^{p-1} \Theta_{X}(k)\right) \rightarrow \ldots$,

where $\lambda$ is the contraction with the hyperplane class in $H^{1}\left(X, \Omega_{X}^{1}\right)$.

Assuming $\omega_{X} \cong \mathcal{O}_{X}(m)$, the nondegenerate pairings $\Omega_{X}^{i} \otimes \Omega_{X}^{n-i} \rightarrow \omega_{X}$ indice isomorphisms $\wedge^{i} \Theta_{X}(m) \cong \Omega_{X}^{n-i}$. Under these isomorphisms, the contraction morphisms

$$
H^{q}\left(X, \bigwedge^{p} \Theta_{X}(m)\right) \stackrel{\lambda}{\rightarrow} H^{q+1}\left(X, \bigwedge^{p-1} \Theta_{X}(m)\right)
$$

become the Lefschetz maps

$$
H^{n-p, q}(X) \stackrel{\lambda}{\rightarrow} H^{n-p+1, q+1}(X) .
$$

Therefor we obtain the following.

Corollary 3.2. Let $X$ be a smooth subcanonical projectively normal variety of dimension $n$, and let $m \in \mathbb{Z}$ be the integer such that $\omega_{X} \cong \mathcal{O}_{X}(m)$. Then we have a long exact sequence

$$
\cdots \rightarrow H^{n-p, q-1}(X) \stackrel{\lambda_{n-p} q-1}{\rightarrow} \rightarrow H^{n-p+1, q}(X) \rightarrow H^{q}\left(U_{X}, \bigwedge^{p} \Theta_{U_{X}}\right)_{m} \rightarrow H^{n-p, q}(X) \stackrel{\lambda_{n-p, q}}{\rightarrow} H^{n-p+1, q+1}(X) \rightarrow \ldots
$$

where $\lambda_{i, j}: H^{i, j}(X) \rightarrow H^{i+1, j+1}(X)$ is the Lefschetz operator. 
We can then prove the following result, expressing the Hochschild cohomology of the puntured cone $U_{X}$ in terms of the primitive cohomology of $X$.

Theorem 3.3. In the above assumptions we have a canonical isomorphism

$$
\mathrm{HH}^{p, q}\left(U_{X}\right)_{m} \cong H_{\mathrm{prim}}^{n-p+1, q}(X) \oplus H_{\mathrm{prim}}^{n-q, p}(X),
$$

where, for each value of $p, q$, at most one of the two summands on the right is nonzero. In particular,

$$
\mathrm{HH}^{p, q}\left(U_{X}\right)_{m} \cong \begin{cases}H_{\mathrm{prim}}^{n-p+1, q}(X) & \text { if } p>q \\ H_{\text {prim }}^{n-q, p}(X) & \text { if } p \leq q .\end{cases}
$$

Proof. The long exact sequence 5 induces the short ones

$$
0 \rightarrow \operatorname{coKer}\left(\lambda_{n-p, q-1}\right) \rightarrow H^{q}\left(U, \bigwedge^{p} \Theta_{U}\right)_{m} \rightarrow \operatorname{Ker}\left(\lambda_{n-p, q}\right) \rightarrow 0 .
$$

If $p \leq q$, then $\operatorname{coKer}\left(\lambda_{n-p, q-1}\right)=0$ and $\operatorname{Ker}\left(\lambda_{n-p, q}\right)=\lambda^{-p+q} H_{\text {prim }}^{n-q, p}(X)$ by Hard Lefschetz, and so

$$
H^{q}\left(U_{X}, \bigwedge^{p} \Theta_{U_{X}}\right)_{m} \cong H_{\text {prim }}^{n-q, p}(X)
$$

in this case. Note that for $p=q$ this gives $H^{p}\left(U_{X}, \wedge^{p} \Theta_{U_{X}}\right)_{m} \cong H_{\text {prim }}^{n-p, p}(X)$.

If $p>q$, again by Hard Lefschetz we have $\operatorname{Ker}\left(\lambda_{n-p, q}\right)=0$ and by definition $\operatorname{coKer}\left(\lambda_{n-p, q-1}\right)=$ $H_{\text {prim }}^{n-p+1, q}(X)$, so that

$$
H^{q}\left(U_{X}, \bigwedge^{p} \Theta_{U_{X}}\right)_{m} \cong H_{\text {prim }}^{n-p+1, q}(X)
$$

in this case. By setting $H_{\text {prim }}^{i, j}(X)=0$ if $i+j>n$, we can summarize the above results as

$$
\mathrm{HH}^{p, q}\left(U_{X}\right)_{m}=H^{q}\left(U_{X}, \bigwedge^{p} \Theta_{U_{X}}\right)_{m} \cong H_{\text {prim }}^{n-p+1, q}(X) \oplus H_{\text {prim }}^{n-q, p}(X)
$$

for any $p, q$.

The above Theorem 3.3 admits a nice rephrasing in terms of the derived deformation complex of $A_{X}$,

$$
T_{A_{X}}^{p, q}:=\operatorname{Ext}_{\mathcal{O}_{A_{X}}}^{q}\left(\wedge^{p} \mathbb{L}_{A_{X}}, \mathcal{O}_{A_{X}}\right) .
$$

Corollary 3.4. Let $X$ be a smooth subcanonical projectively normal variety of dimension $n$ which is arithmetically Cohen-Macaulay, and let $m \in \mathbb{Z}$ be the integer such that $\omega_{X} \cong$ $\mathcal{O}_{X}(m)$. Let $A_{X}$ the affine cone of $X$. Then, for every $1 \leq p \leq n+1$ and $0 \leq q \leq n$, we have

$$
\left(T_{A_{X}}^{p, q}\right)_{m}=\operatorname{Ext}_{\mathcal{O}_{A_{X}}}^{q}\left(\Omega_{A_{X}}^{p}, \mathcal{O}_{A_{X}}\right)_{m} \cong\left\{\begin{array}{l}
H_{\mathrm{prim}}^{n-p+1, q}(X) \text { if } p>q \\
H_{\mathrm{prim}}^{n-q, p}(X) \text { if } p \leq q .
\end{array}\right.
$$


Proof. Since $X$ is a smooth projective variety of dimension $n$, the affine cone $A_{X}$ is smooth in codimension $n+1$ and so for $1 \leq p \leq n+1$ and $0 \leq q \leq n$ we have $T_{A_{X}}^{p, q}=\operatorname{Ext}_{\mathcal{O}_{A_{X}}}^{q}\left(\Omega_{A_{X}}^{p}, \mathcal{O}_{A_{X}}\right)$, see, e.g., [17, Lemma 3.2]. Since $X$ is also arithmetically Cohen-Macaulay, we have $\operatorname{depth}_{0} A_{X} \geq n$ and this, following SGA 2 Exposè VI, [22], implies that the inclusion $U_{X} \hookrightarrow A_{X}$ induces an isomorphism $\operatorname{Ext}_{\mathcal{O}_{A_{X}}}^{q}\left(\Omega_{A_{X}}^{p}, \mathcal{O}_{A_{X}}\right) \cong$ $\operatorname{Ext}_{\mathcal{O}_{U_{X}}}^{q}\left(\Omega_{U_{X}}^{p}, \mathcal{O}_{U_{X}}\right)$. Finally, since $U_{X}$ is smooth, we have $\operatorname{Ext}_{\mathcal{O}_{U_{X}}}^{q}\left(\Omega_{U_{X}}^{p}, \mathcal{O}_{U_{X}}\right) \cong \operatorname{HH}^{p, q}\left(U_{X}\right)$.

Although the above corollary is essentially a rephrasing of Theorem 3.3, it is important to stress that, when we consider the whole affine cone, the Ext modules becomes easy to compute using computer algebra software as SINGULAR or MACAULAY2 ([16], [20]). In particular it should be possible to write down a computer package - similar to the ne already existing for $T^{1}$ and $T^{2}$ - able to compute all of the Hodge numbers of a smooth projective arithmetically Cohen-Macaulay variety.

3.3. The case of a hypersurface. The results of the previous section lead to an interesting corollary in the case of a hypersurface: we can use them to recover Griffiths' isomorphism between the primitive cohomology of a degree $d$ smooth hypersurface $X \subseteq \mathbb{P}^{n+1}$ and a distinguished graded component of the Milnor algebra of a polynomial defining $X$. We start with some preliminary Lemmas. Most of the proofs are a straightforward consequence of Lemma 3.1, of the short exact sequences

$$
\left.0 \rightarrow \bigwedge^{p-i} \Theta_{X} \rightarrow \bigwedge^{p-i} \Theta_{\mathbb{P}^{n+1}}\right|_{X} \rightarrow \bigwedge^{p-i-1} \Theta_{X}(d) \rightarrow 0
$$

and

$$
\left.0 \rightarrow \Omega_{\mathbb{P}^{n+1}}^{k}(-d) \rightarrow \Omega_{\mathbb{P}^{n+1}}^{k} \rightarrow \Omega_{\mathbb{P}^{n+1}}^{k}\right|_{X} \rightarrow 0,
$$

of the duality isomorphisms

$$
\bigwedge^{i} \Theta_{X}(d-n-2) \cong \Omega_{X}^{n-i}
$$

and

$$
\left.\left.\bigwedge^{i} \Theta_{\mathbb{P}^{n+1}}\right|_{X}(-n-2) \cong \Omega_{\mathbb{P}^{n+1}}^{n+1-i}\right|_{X}
$$

and of the Kodaira and Bott vanishing theorems [28] and are therefore omitted. The proof of Lemma 3.8 is a bit more subtle, so it is spelled out in full detail. It also serves as an exemplification of the technique used to prove all the other Lemmas in this section.

Lemma 3.5. For $X$ a smooth, projective hypersurface in $\mathbb{P}^{n+1}$ of degree $d$, we have a natural isomorphism

$$
H^{p-i}\left(U_{X}, \bigwedge^{p-i} \Theta_{U_{X}}\right)_{m+k d} \cong H^{p-i}\left(X, \bigwedge^{p-i} \Theta_{X}(m+k d)\right),
$$

where $m=d-n-2$, for every $0 \leq i \leq p \leq n$ and every $k \geq 1$. In particular, for every $p \geq 2$ we have natural isomorphisms

$$
H^{1}\left(U_{X}, \Theta_{U_{X}}\right)_{m+(p-1) d} \cong H^{1}\left(X, \Theta_{X}(m+(p-1) d)\right)
$$


and

$$
H^{p-1}\left(U_{X}, \bigwedge^{p-1} \Theta_{U_{X}}\right)_{m+d} \cong H^{p-1}\left(X, \bigwedge^{p-1} \Theta_{X}(m+d)\right)
$$

Lemma 3.6. Let $X$ a smooth, projective hypersurface in $\mathbb{P}^{n+1}$ of degree d. Then we have a natural isomorphism

$$
H^{p-i-1}\left(X, \bigwedge^{p-i-1} \Theta_{X}(m+(k+1) d)\right) \cong H^{p-i}\left(X, \bigwedge^{p-i} \Theta_{X}(m+k d)\right),
$$

where $m=d-n-2$, for every $0 \leq i \leq p-2$, with $0 \leq p \leq n$. In particular, one has a natural isomorphism

$$
H^{1}\left(X, \Theta_{X}(m+(p-1) d)\right) \cong H^{p-1}\left(X, \bigwedge^{p-1} \Theta_{X}(m+d)\right)
$$

for any $2 \leq p \leq n$.

Lemma 3.7. Let $0 \leq p \leq n$. If $n \neq 2 p$ then we have a natural isomorphism

$$
H^{p}\left(U_{X}, \bigwedge^{p} \Theta_{U_{X}}\right)_{m} \cong H^{p}\left(X, \bigwedge^{p} \Theta_{X}(m)\right)
$$

Proof. We know from Theorem 3.3 that $H^{p}\left(U_{X}, \wedge^{p} \Theta_{U_{X}}\right)_{m} \cong H_{\text {prim }}^{n-p, p}(X)$. But for a smooth hypersurface in $\mathbb{P}^{n+1}$ one has $H^{p, n-p}(X)=H_{\text {prim }}^{p, n-p}(X)$ for any $p$ such $n \neq 2 p$, due to Hard Lefschetz combined with the Lefschetz hyperplane theorem.

Lemma 3.8. Let $0 \leq p \leq n$. If $n \neq 2 p$ then we have a natural isomorphism

$$
H^{p-1}\left(\bigwedge^{p-1} \Theta_{X}(m+d)\right) \cong H^{p}\left(\bigwedge^{p} \Theta_{X}(m)\right)
$$

Proof. From the short exact sequence

$$
\left.0 \rightarrow \bigwedge^{p} \Theta_{X} \rightarrow \bigwedge^{p} \Theta_{\mathbb{P}^{n+1}}\right|_{X} \rightarrow \bigwedge^{p-1} \Theta_{X}(d) \rightarrow 0
$$

using the duality isomorphisms $\bigwedge^{i} \Theta_{X}(m) \cong \Omega_{X}^{n-i}$ and $\left.\left.\bigwedge^{i} \Theta_{\mathbb{P}^{n+1}}\right|_{X}(m) \cong \Omega_{\mathbb{P}^{n+1}}^{n+1-i}\right|_{X}(d)$ we get to the long exact sequence

$$
\begin{gathered}
\ldots \rightarrow H^{n-p, p-1}(X) \rightarrow H^{p-1}\left(X,\left.\Omega_{\mathbb{P}^{n+1}}^{n-p+1}(d)\right|_{X}\right) \rightarrow H^{p-1}\left(X, \Omega_{X}^{n-p+1}(d)\right) \rightarrow \\
\rightarrow H^{n-p, p}(X) \rightarrow H^{p}\left(X,\left.\Omega_{\mathbb{P}^{n+1}}^{n-p+1}(d)\right|_{X}\right) \rightarrow 0,
\end{gathered}
$$

where the last zero comes from $H^{p}\left(X, \Omega_{X}^{n-p+1}(d)\right)=0$ by Kodaira Vanishing. Now, consider the short exact sequence (see [7])

$$
\left.0 \rightarrow \Omega_{\mathbb{P}^{n+1}}^{n-p+1} \rightarrow \Omega_{\mathbb{P}^{n+1}}^{n-p+1}(d) \rightarrow \Omega_{\mathbb{P}^{n+1}}^{n-p+1}(d)\right|_{X} \rightarrow 0 .
$$

This induces the long exact sequence

$$
\begin{aligned}
& \cdots \rightarrow H^{p}\left(\mathbb{P}^{n+1}, \Omega_{\mathbb{P}^{n+1}}^{n-p+1}(d)\right) \rightarrow H^{p}\left(\mathbb{P}^{n+1},\left.\Omega_{\mathbb{P}^{n+1}}^{n-p+1}(d)\right|_{X}\right) \rightarrow \\
& \rightarrow H^{p+1}\left(\mathbb{P}^{n+1}, \Omega_{\mathbb{P}^{n+1}}^{n-p+1}\right) \rightarrow H^{p+1}\left(\mathbb{P}^{n+1}, \Omega_{\mathbb{P}^{n+1}}^{n-p+1}(d)\right) \rightarrow \cdots
\end{aligned}
$$


By Kodaira vanishing we have $H^{p+1}\left(\mathbb{P}^{n+1}, \Omega_{\mathbb{P}^{n+1}}^{n-p+1}(d)\right)=0$. Also we have the long exact sequence

$$
\begin{aligned}
\cdots \rightarrow & H^{p-1}\left(\mathbb{P}^{n+1}, \Omega_{\mathbb{P}^{n+1}}^{n-p+1}(d)\right) \rightarrow H^{p-1}\left(\mathbb{P}^{n+1},\left.\Omega_{\mathbb{P}^{n+1}}^{n-p+1}(d)\right|_{X}\right) \rightarrow \\
& \rightarrow H^{p}\left(\mathbb{P}^{n+1}, \Omega_{\mathbb{P}^{n+1}}^{n-p+1}\right) \rightarrow H^{p}\left(\mathbb{P}^{n+1}, \Omega_{\mathbb{P}^{n+1}}^{n-p+1}(d)\right) \rightarrow \cdots
\end{aligned}
$$

By Bott vanishing also

and

$$
H^{p}\left(\mathbb{P}^{n+1}, \Omega_{\mathbb{P}^{n+1}}^{n-p+1}(d)\right)=0
$$

$$
H^{p-1}\left(\mathbb{P}^{n+1}, \Omega_{\mathbb{P} n+1}^{n-p+1}(d)\right)=0 .
$$

Now we consider two subcases. If $n \neq 2 p-1$, then

$$
H^{p}\left(\mathbb{P}^{n+1},\left.\Omega_{\mathbb{P}^{n+1}}^{n-p+1}(d)\right|_{X}\right) \cong H^{p+1}\left(\mathbb{P}^{n+1}, \Omega_{\mathbb{P}^{n+1}}^{n-p+1}\right)=0
$$

and

$$
H^{p-1}\left(\mathbb{P}^{n+1},\left.\Omega_{\mathbb{P}^{n+1}}^{n-p+1}(d)\right|_{X}\right) \cong H^{p}\left(\mathbb{P}^{n+1}, \Omega_{\mathbb{P}^{n+1}}^{n-p+1}\right)=0
$$

(where we used that by hypothesis $n \neq 2 p$ ). So, we find

$$
H^{p-1}\left(X, \Omega_{X}^{n-p+1}(d)\right) \cong H^{p}\left(X, \Omega_{X}^{n-p}\right)
$$

i.e.,

$$
H^{p-1}\left(X, \bigwedge^{p-1} \Theta_{X}(m+d)\right) \cong H^{p}\left(X, \bigwedge^{p} \Theta_{X}(m)\right)
$$

in this case. If $n=2 p-1$ then we still have

$$
H^{p}\left(X,\left.\Omega_{\mathbb{P}^{2 p}}^{p}(d)\right|_{X}\right)=H^{p}\left(\mathbb{P}^{2 p},\left.\Omega_{\mathbb{P}^{2 p}}^{p}(d)\right|_{X}\right) \cong H^{p+1}\left(\mathbb{P}^{2 p}, \Omega_{\mathbb{P}^{2 p}}^{p}\right)=0
$$

while

$$
H^{p-1}\left(X,\left.\Omega_{\mathbb{P}^{2 p}}^{p}(d)\right|_{X}\right)=H^{p-1}\left(\mathbb{P}^{2 p},\left.\Omega_{\mathbb{P}^{2 p}}^{p}(d)\right|_{X}\right) \cong H^{p}\left(\mathbb{P}^{2 p}, \Omega_{\mathbb{P}^{2 p}}^{p}\right) \cong \mathbb{C} .
$$

and so our initial long exact sequence becomes

$$
\ldots \rightarrow H^{p-1, p-1}(X) \stackrel{\eta}{\rightarrow} H^{p, p}\left(\mathbb{P}^{2 p}\right) \rightarrow H^{p-1}\left(X, \Omega_{X}^{p}(d)\right) \rightarrow H^{p-1, p}(X) \rightarrow 0 .
$$

By precomposing the map $\eta$ with the restriction morphism

$$
H^{p-1, p-1}\left(\mathbb{P}^{2 p}\right) \rightarrow H^{p-1, p-1}(X)
$$

one obtains the cup product with $c_{1}\left(\mathcal{O}_{\mathbb{P}^{2 p}}(d)\right)$, which is an isomorphism from

$$
H^{p-1, p-1}\left(\mathbb{P}^{2 p}\right) \rightarrow H^{p, p}\left(\mathbb{P}^{2 p}\right) .
$$

Hence $\eta$ is surjective, and so

$$
H^{p-1}\left(X, \Omega_{X}^{p}(d)\right) \cong H^{p-1, p}(X) .
$$

Therefore,

in this case, too.

$$
H^{p-1}\left(X, \bigwedge^{p-1} \Theta_{X}(m+d)\right) \cong H^{p}\left(X, \bigwedge^{p} \Theta_{X}(m)\right)
$$

Corollary 3.9. For any $2 \leq p \leq n$ with $n \neq 2 p$ one has a natural isomorphism

$$
H^{1}\left(U_{X}, \Theta_{U_{X}}\right)_{m+(p-1) d} \cong H^{p}\left(U_{X}, \bigwedge^{p} \Theta_{U_{X}}\right)_{m}
$$


We are now left with considering the $n=2 p$ case.

Lemma 3.10. Assume $n=2 p$. Then we have a natural short exact sequence

$$
0 \rightarrow H^{p-1}\left(X, \bigwedge^{p-1} \Theta_{X}(m+d)\right) \rightarrow H^{p, p}(X) \rightarrow \mathbb{C} \rightarrow 0,
$$

Proof. Reasoning as in the proof of 3.7 we get the long exact sequence

$$
\ldots \rightarrow H^{p-1}\left(X,\left.\Omega_{\mathbb{P}^{2 p+1}}^{p+1}(d)\right|_{X}\right) \rightarrow H^{p-1}\left(X, \Omega_{X}^{p+1}(d)\right) \rightarrow H^{p, p}(X) \rightarrow H^{p}\left(X,\left.\Omega_{\mathbb{P}^{n+1}}^{p+1}(d)\right|_{X}\right) \rightarrow 0,
$$

and we have

$$
H^{p-1}\left(\mathbb{P}^{2 p+1},\left.\Omega_{\mathbb{P}^{2 p+1}}^{p+1}(d)\right|_{X}\right) \cong H^{p}\left(\mathbb{P}^{2 p+1}, \Omega_{\mathbb{P}^{2 p+1}}^{p+1}\right)=0
$$

and

$$
H^{p}\left(\mathbb{P}^{2 p+1},\left.\Omega_{\mathbb{P}^{2 p+1}}^{p+1}(d)\right|_{X}\right) \cong H^{p+1}\left(\mathbb{P}^{2 p+1}, \Omega_{\mathbb{P}^{2 p+1}}^{p+1}\right) \cong \mathbb{C}
$$

Corollary 3.11. Assume $n=2 p$, with $p \geq 2$. Then there exists an isomorphsim

$$
H^{1}\left(U_{X}, \Theta_{U_{X}}\right)_{m+(p-1) d} \cong H^{p}\left(U_{X}, \bigwedge^{p} \Theta_{U_{X}}\right)_{m}
$$

Proof. By Lemmas 3.5 and 3.6 we have an isomorphism

$$
H^{1}\left(U_{X}, \Theta_{U_{X}}\right)_{m+(p-1) d} \cong H^{p-1}\left(U_{X}, \bigwedge^{p-1} \Theta_{U_{X}}\right)_{m+d},
$$

so we need only to exhibit an isomorphism

$$
H^{p-1}\left(U_{X}, \bigwedge^{p-1} \Theta_{U_{X}}\right)_{m+d} \cong H^{p}\left(U_{X}, \bigwedge^{p} \Theta_{U_{X}}\right)_{m}
$$

To do this, recall the isomorphism $H^{p}\left(U_{X}, \wedge^{p} \Theta_{U_{X}}\right)_{m} \cong H_{\text {prim }}^{p, p}(X)$ from 3.3, the short exact sequence

$$
0 \rightarrow H_{\text {prim }}^{p, p}(X) \rightarrow H^{p, p}(X) \stackrel{\lambda}{\rightarrow} H^{p+1, p+1}(X) \rightarrow 0
$$

coming from Hard Lefschetz, and the fact that $H^{p+1, p+1}(X) \cong \mathbb{C}$ from the Lefschetz hyperplane theorem combined with Hard Lefschetz. Therefore we have a natural short exact sequence

$$
0 \rightarrow H^{p}\left(U_{X}, \bigwedge^{p} \Theta_{U_{X}}\right)_{m} \rightarrow H^{p, p}(X) \rightarrow \mathbb{C} \rightarrow 0,
$$

and we use Lemma 3.10 and Lemma 3.5 to conclude.

Putting all the above results toghether we obtain the following

Theorem 3.12. Let $X \subseteq \mathbb{P}^{n+1}$ be a smooth degree d projective hypersurface, with $\operatorname{dim} X=$ $n \geq 3$. Then we have

$$
H^{1}\left(U_{X}, \Theta_{U_{X}}\right)_{p d-n-2} \cong H^{p}\left(U_{X}, \bigwedge^{p} \Theta_{U_{X}}\right)_{d-n-2}
$$

for every $0 \leq p \leq \operatorname{dim} X$. 
Proof. Since $d-n-2$ is precisely the integer $m$ such that $\omega_{X} \cong \mathcal{O}_{X}(m)$ by adjunction, for $p \geq 2$ the result follows from Corollary 3.11 and Corollary 3.9. For $p=1$ there is nothing to prove. Finally, for $p=0$ we have to show that

$$
H^{1}\left(U_{X}, \Theta_{U_{X}}\right)_{-n-2} \cong H^{0}\left(U_{X}, \mathcal{O}_{U_{X}}\right)_{d-n-2}
$$

On the right hand side we have $H^{0}\left(X, \mathcal{O}_{X}(d-n-2)\right)$, while on the left hand side we consider the short exact sequence

$$
0 \rightarrow \operatorname{coker}\left\{\lambda_{n-1,0}(-d)\right\} \rightarrow H^{1}\left(U_{X}, \Theta_{U_{X}}\right)_{-n-2} \rightarrow \operatorname{ker}\left\{\lambda_{n-1,1}(-d)\right\} \rightarrow 0
$$

where $\lambda_{i, j}(-d)$ is the Lefschetz morphism

$$
H^{j}\left(X, \Omega_{X}^{i}(-d)\right) \rightarrow H^{j+1}\left(X, \Omega_{X}^{i+1}(-d)\right) .
$$

Now we have

$$
H^{1}\left(X, \Omega_{X}^{n}(-d)\right)=H^{1}(X, \mathcal{O}(-n-2))=0
$$

and

$$
H^{2}\left(X, \Omega_{X}^{n}(-d)\right)=H^{2}\left(X, \mathcal{O}_{X}(-n-2)\right)=0,
$$

since $X$ is arithmetically Cohen-Macaulay. So the above short exact sequence gives

$$
H^{1}\left(U_{X}, \Theta_{U_{X}}\right)_{-n-2} \cong H^{1}\left(X, \Theta_{X}(-n-2)\right)
$$

and to conclude the proof of the theorem we only need to show that

$$
H^{1}\left(X, \Theta_{X}(-n-2)\right) \cong H^{0}\left(X, \mathcal{O}_{X}(d-n-2)\right) .
$$

This follows from the normal sheaf exact sequence as in the proof of Lemma 3.6.

Now, why is this interesting? Thanks to the computation above, we have

$$
H_{\text {prim }}^{p, n-p}(X) \cong H^{p}\left(U_{X}, \bigwedge^{p} \Theta_{U_{X}}\right)_{m} \cong H^{1}\left(U_{X}, \Theta_{U_{X}}\right)_{p d-n-2}
$$

On the other hand we know that for a degree $d$ smooth projective hypersurface defined by the polynomial $f$ we have

$$
H^{1}\left(U_{X}, \Theta_{U_{X}}\right) \cong T_{A_{X}}^{1} \cong \mathcal{M}_{f}[d] .
$$

Therefore, we recover the following result from Griffiths' residue theory [21].

Corollary 3.13. Let $X \subseteq \mathbb{P}^{n+1}$ be a degree $d$ smooth projective hypersurface with $\operatorname{dim} X \geq$ 3 , defined by the polynomial $f$. Then we have

$$
\left(\mathcal{M}_{f}[d]\right)_{p d-n-2} \cong H_{\text {prim }}^{p, n-p}(X)
$$

for every $0 \leq p \leq \operatorname{dim} X$. Equivalently, one has

$$
\left(\mathcal{M}_{f}\right)_{p d-n-2} \cong H_{\mathrm{prim}}^{p-1, n-p+1}(X)
$$

for every $1 \leq p \leq \operatorname{dim} X+1$. 


\section{REFERENCES}

[1] E. Arbarello and E. Sernesi. Petri's approach to the study of the ideal associated to a special divisor. Invent. Math., 49(2):99-119, 1978.

[2] M. F. Atiyah. Complex analytic connections in fibre bundles. Trans. Amer. Math. Soc., 85:181-207, 1957.

[3] S. A. Barannikov. Extended moduli spaces and mirror symmetry in dimensions $n>3$. ProQuest LLC, Ann Arbor, MI, 1999. Thesis (Ph.D.)-University of California, Berkeley.

[4] V. Batyrev, I. Ciocan-Fontanine, B. Kim, and D. Van Straten. Conifold transitions and mirror symmetry for Calabi-Yau complete intersections in Grassmannians Nuclear Phys. B., 514: 640-666, 1998.

[5] K. Behrend and B. Noohi. Moduli of non-commutative polarized schemes. ArXiv e-prints, July 2015.

[6] Y. Bespalov, V. Lyubashenko, and O. Manzyuk. Pretriangulated $A_{\infty}$-categories. Pratsi Instytutu Matematyky Natsional'noï Akademiï Nauk Ukraïny. Matematyka ta ïi Zastosuvannya 76. Kyïv: Instytut Matematyky NAN Ukraïny. 598 p. , 2008.

[7] I. Biswas and G. Schumacher. On the stability of the tangent bundle of a hypersurface in a Fano variety. J. Math. Kyoto Univ., 45(4):851-860, 2005.

[8] G. E. Bredon. Sheaf theory. Graduate Text in Mathematics, 170, Springer-Verlag New York, 1967, XI,504.

[9] G. Brown, A. M. Kasprzyk, and al. Graded ring database. http://grdb.co.uk, 2015.

[10] R.-O. Buchweitz and H. Flenner. The global decomposition theorem for Hochschild (co-)homology of singular spaces via the Atiyah-Chern character. Adv. Math., 217(1):243-281, 2008.

[11] L. Bădescu. Projective Geometry and Formal Geometry. Monografie Matematyczne, 65, Birkhäuser Basel, XIV,214.

[12] A. Căldăraru. The Mukai pairing. II. The Hochschild-Kostant-Rosenberg isomorphism. Adv. Math., 194(1):34-66, 2005.

[13] J. Carlson, S. Mueller-Stach, and C. Peters. Period Mappings and Period Domains. Cambridge Studies in Avdanced Mathematics, 2003.

[14] P. De Poi and F. Zucconi. On subcanonical Gorenstein varieties and apolarity. J. Lond. Math. Soc. (2), 87(3):819-836, 2013.

[15] O. Debarre, A. Iliev, and L. Manivel. On the period map for prime Fano threefolds of degree 10. J. Algebraic Geom., 21(1):21-59, 2012.

[16] W. Decker, G.-M. Greuel, G. Pfister, and H. Schönemann. Singular 4-0-2 - A computer algebra system for polynomial computations. http://www.singular.uni-kl.de 2015.

[17] M. Filip. Hochschild cohomology and deformation quantisation of affine topic varieties arXiv: $1706.00580 \mathrm{v} 1$

[18] M. Fiorentini and A. Lascu. On the homogeneous ideal of a quasi-complete intersection in the projective space. Annali dell'Università di Ferrara, 29(1):211-219, 1983.

[19] W. Fulton and J. Harris. Representation Theory: A First Course. Graduate Texts in Mathematics / Readings in Mathematics. Springer New York, 1991.

[20] D. R. Grayson and M. E. Stillman. Macaulay2, a software system for research in algebraic geometry. http://www.math.uiuc.edu/Macaulay2/

[21] P. Griffiths. Periods of integrals on algebraic manifolds, i, ii, iii. American Journal of Mathematics, 90(3):805-865, 1968.

[22] A. Grothendieck. Cohomologie locale des faisceaux cohérents... (SGA 2), volume 2 of Advanced Studies in Pure Mathematics. North-Holland Publishing Co., 1968.

[23] F. Hirzebruch. Topological Methods in Algebraic Geometry. Springer-Verlag Berlin Heidelberg, 1978.

[24] N. M. Katz and T. Oda. On the differentiation of De Rham cohomology classes with respect to parameters. J. Math. Kyoto Univ., 8(2):199-213, 1968.

[25] S. Kebekus, T. Peternell, A. J. Sommese, and J. A. Wiśniewski. Projective contact manifolds. Inventiones mathematicae, 142(1):1-15, 2000. 
[26] A. Kanazawa. Pfaffian Calabi-Yau threefolds and mirror symmetry. Commun. Number Theory Phys. 6 (2012), no. 3, 661-696.

[27] M. Kontsevich. Homological algebra of mirror symmetry. In Proceedings of the International Congress of Mathematicians, Vol. 1, 2 (Zürich, 1994), pages 120-139. Birkhäuser, Basel, 1995.

[28] E. N. Materov. The Bott formula for toric varieties. Mosc. Math. J., 2(1):161-182, 200, 2002.

[29] S. Mukai. Curves and Grassmannians. In Algebraic geometry and related topics (Inchon, 1992), Conf. Proc. Lecture Notes Algebraic Geom., I, pages 19-40. Int. Press, Cambridge, MA, 1993.

[30] M. Schlessinger. Functors of artin rings. Transactions of the American Mathematical Society, 130:208$222,1968$.

[31] M. Schlessinger. On rigid singularities. The Rice University Studies, 19(1):147-162, 1973.

[32] M. Schlessinger and S. Lichtenbaum. The cotangent complex of a morphism. Transactions of the American Mathematical Society,128.1 (1967): 41-70.

[33] F. Schuhmacher. Hochschild cohomology of complex spaces and Noetherian schemes. Homology, Homotopy and Applications, 6.1 (2004): 299-340.

[34] E. Sernesi. Deformation of Algebraic Schemes. Grundlehren der Mathematischen Wisschenschaften [Fundamental Principles of Mathematical Sciences], 334, Springer-Verlag, Berlin, 2006. xii+339 pp.

[35] C. Voisin. Hodge Theory and Complex Algebraic Geometry, II. Cambridge Studies in Advanced Mathematics (No. 76), 2002.

[36] J. Wahl. The Jacobian algebra of a graded Gorenstein singularity. Duke Mathematical Journal, $55(4): 843-871,1987$.

King's College London, Floor 5, Waterloo Bridge Wing, Franklin Wilkins Building,, 150 Stamford Street, London SE1 9NH, United Kingdom

E-mail address, C. Di Natale: carmelo.di_natale@kcl.ac.uk

Mathematics Institute, Zeeman Building, University of Warwick, Coventry CV4 7AL, UNITED KingDOM

E-mail address, E. Fatighenti: E.Fatighenti@warwick.ac.uk

Dipartimento di Matematica "Guido Castelnuovo", Università degli Studi di Roma "La Sapienza", Piazzale Aldo Moro, 2, 00185 Roma, Italy

E-mail address, D. Fiorenza: fiorenza@mat.uniroma1.it 